\title{
Protective Effect of Yinhua Miyanling Tablet on Lipopolysaccharide-Induced Inflammation through Suppression of NLRP3/Caspase-1 Inflammasome in Human Peripheral Blood Mononuclear Cells
}

\author{
Jingying Sai, ${ }^{1,2}$ Lingxin Xiong, ${ }^{1,3}$ Jingtong Zheng, ${ }^{1}$ Chuangui Liu, ${ }^{3}$ Yanjiao Lu, ${ }^{1}$ \\ Guoqiang Wang, ${ }^{1}$ Yawei Wang, ${ }^{1}$ Ting Wang, ${ }^{1}$ Xuewa Guan, ${ }^{1}$ Fang Chen, ${ }^{1}$ Keyong Fang, \\ Chao Zhang, ${ }^{1}$ Junying Lu, ${ }^{1}$ Xiaotian Zhang, ${ }^{1}$ Hailin Zhu, ${ }^{3}$ and Fang Wang ${ }^{1}$ \\ ${ }^{1}$ Department of Pathogeny Biology, College of Basic Medical Sciences, Jilin University, Changchun 130021, China \\ ${ }^{2}$ Department of Clinical Laboratory, The Second Hospital of Jilin University, Changchun 130021, China \\ ${ }^{3}$ School of Pharmaceutical Sciences, Jilin University, Changchun 130021, China
}

Correspondence should be addressed to Fang Wang; wf@jlu.edu.cn

Received 2 March 2016; Revised 30 June 2016; Accepted 13 July 2016

Academic Editor: Hyunsu Bae

Copyright (C) 2016 Jingying Sai et al. This is an open access article distributed under the Creative Commons Attribution License, which permits unrestricted use, distribution, and reproduction in any medium, provided the original work is properly cited.

\begin{abstract}
Yinhua Miyanling Tablet (YMT), the Chinese formula, has long been administrated in clinical practice for the treatment of acute pyelonephritis and acute urocystitis. In the current study, we aimed to investigate the anti-inflammatory effect of YMT in vitro and to evaluate the association between anti-inflammation and innate immune response. Human peripheral blood mononuclear cells (PBMCs) were isolated using Ficoll density gradient centrifugation and then were stimulated by Lipopolysaccharide (LPS). The differential gene expression of inflammation-related genes after drug administration was assessed using PCR array, and the protein levels of differential genes were measured by ELISA and Western blot. The result showed that YMT significantly inhibited the expression of NLRP3, Caspase-1, and the downstream cytokine IL- $1 \beta$ and suppressed the production of inflammatory mediators TNF- $\alpha$, IL-6, IL-10, and MCP-1 in a dose-dependent manner compared to the LPS group $(P<0.01)$. The finding indicated that YMT exhibited anti-inflammatory effect in vitro by suppressing the NLRP3/Caspase-1 inflammasome, and that may have therapeutic potential for the treatment of inflammatory diseases.
\end{abstract}

\section{Introduction}

Traditional Chinese medicine (TCM), guided by the theory of traditional Chinese medical science, has been applied for over 5,000 years and is valued for its multitarget and less side-effect properties [1]. Yinhua Miyanling Tablet (YMT) formula is composed of ten traditional Chinese medicines: Lonicerae Japonicae Flos, Scutellariae Barbatae Herba, Polygoni Avicularis Herba, Dianthi Herba, Pyrrosiae Folium, Clematidis Armandii Caulis, Plantaginis Semen, Lophatheri Herba, Taxilli Herba, and Junci Medulla [2]. YMT has been widely used in acute pyelonephritis, acute urocystitis, and chronic prostatitis for clearing heat and detoxifying and for removing dampness through diuresis for more than a decade
$[3,4]$. Previous studies reported that phenol extract of Flos Lonicerae, the monarch drug in the prescription, exhibited significant antibacterial and anti-inflammatory activities [511]. Jin et al. found that phenol extract of Flos Lonicerae significantly inhibited inflammatory cell infiltration and protein accumulation and inhibited the production of TNF- $\alpha$, PEG2, and NO in Lipopolysaccharide- (LPS-) induced aqueous humor in rat [12]. Alcohol extract of Flos Lonicerae promotes the repair of incision and effectively inhibits the secretion of TNF- $\alpha$ and IL- 6 and significantly increases the production of IL-10 [13]. However, the anti-inflammatory effect of YMT in vitro was not well studied. In addition, despite rich clinical experience, the lack of research in molecular mechanism of action weakens the scientific validity of YMT. Thus, we 
investigated the anti-inflammatory effect of YMT in vitro using modern research methods, including PCR array.

When inflammatory stimulus occurs, innate immunity, as the first defender, recognizes and removes invading microorganisms [14, 15]. Therefore, we hypothesized that innate immunity was involved in the anti-inflammatory effect of YMT. NLRP3 (NOD-LRRs containing pyrin domain 3, also known as cryopyrin or NALP3) inflammasome, including NLRP3, ASC, and Caspase-1, is the most studied complex that is involved in innate immunity. PRR (Pattern Recognition Receptor), expressed in the surface of innate immune cells, recognizes PAMP (Pathogen Associated Molecular Pattern) or DAMP (Damage-Associated Molecular Patterns), then initiates effective innate immune responses, and eventually clears the microorganisms and endogenous molecules released by the host. Inactive pro-Caspase- 1 can itself be activated by the NLRP3 inflammasome upon pathogen and damage-associated danger signals; active Caspase-1 degrades pro-IL- $1 \beta$ and pro-IL-18, the precursor form of IL- $1 \beta$ and IL18 , into the mature cytokines by proteolysis, which initiates the secretion of the cytokines.

In the current study, we aimed to investigate the antiinflammatory effect of YMT in vitro and to evaluate whether YMT had anti-inflammatory effect that was mediated by the regulation of innate immunity.

\section{Materials and Methods}

2.1. Test Article and Treatment. Yinhua Miyanling Tablet (YMT) was provided by Jilin Huakang Pharmaceutical Co., Ltd. (Lot number: 140306). Some standard compounds were also used in this study. Chlorogenic acid (Lot number: 110753201415), Rutin (100080-201408), Hyperoside (111521-201205), Naringin (110722-201312), geniposidic acid (111828-201403), Scutellarin (110842-201508), Luteolin (111520-200504), luteoloside (111720-201408), and Apigenin (111901-201102) were purchased from the National Institutes for Food and Drug Control (Beijing, China). Scutellarein (Lot number: 141128) was purchased from the Sichuan Weikeqi Biological Technology Co., Ltd.

The fingerprint of YMT was further analyzed with a high performance liquid chromatography (HPLC) system (Waters, USA) that consisted of a model 1525 Waters pump, model 2998 Photodiode Array Detector, and Diamonsil C18 Column $(5 \mu \mathrm{m}, 250 \mathrm{~mm} \times 4.6 \mathrm{~mm})$. The mobile phase was comprised of acetonitrile (A) and $1 \%$ acetic acid in water solvent (B). The gradient mode was as follows: initial 5\% A linear gradient to $15 \% \mathrm{~A}$ in $15 \mathrm{~min}$; linear gradient to $17 \% \mathrm{~A}$ in $35 \mathrm{~min}$; linear gradient to $20 \% \mathrm{~A}$ in $55 \mathrm{~min}$; linear gradient to $55 \% \mathrm{~A}$ in $80 \mathrm{~min}$. The flow rate was $1.0 \mathrm{~mL} / \mathrm{min}$. The components of YMT were identified by the comparison of the retention time from the chromatograms with those known standards. The fingerprints of the mixed standard compounds and the extract of YMT were shown in Figures 1 and 2.

2.2. Materials. LPS and pancreatic enzymes were purchased from Sigma-Aldrich (St. Louis, MO, USA); lymphocyte separation medium was purchased from Jingyang Biological
(Tianjin, China); fetal bovine serum (FBS) was obtained from GIBCO (Australia); RPMI-1640 glucose free medium was purchased from Hyclone (Logan, UT, USA); RNeasy Mini Kit, RNase-Free DNase Set, RT ${ }^{2}$ First Strand Kit, RT ${ }^{2}$ SYBR $^{\circledR}$ Green ROX qPCR Mastermix, and Human Inflammasomes PCR Array were obtained from Qiagen (Valencia, CA, USA). ELISA kits of human TNF- $\alpha$, IL-1 $\beta$, IL-6, IL-10, and MCP1 were purchased from RayBiotech (Atlanta, USA). AntiNLRP3 and anti-Caspase-1 antibodies were purchased from Abcam (Cambridge, United Kingdom). Secondary antibodies were obtained from Sigma-Aldrich (St. Louis, MO, USA).

2.3. Cell Treatment. PBMC was isolated from heparin anticoagulation venous blood via Ficoll density gradient centrifugation method. Then cell viability of isolated PBMC was measured by trypan blue exclusion assay. $50 \mu \mathrm{L}$ of the cell suspension was mixed with $50 \mu \mathrm{L}$ of $0.2 \%$ trypan blue and incubated for $5 \mathrm{~min}$ at room temperature. $10 \mu \mathrm{L}$ of this mixture was injected beneath the cover slip. The unstained (viable) and stained (dead) cells were counted in a total of 200 cells under microscope (Olympus, Japan). The cell viability was calculated according to the following formula: cell viability $=$ total number of viable cells/(total number of viable cells + total number of dead cells) $* 100 \%$. Cells were cultured in RPMI-1640 culture medium containing 10\% FBS in a $37^{\circ} \mathrm{C}$ incubator in the presence of $5 \% \mathrm{CO}_{2}$. The cells were divided into 5 groups including blank control group (control), LPS-stimulated group (LPS), and three YMT-treated groups including a high concentration group $(0.75 \mathrm{mg} / \mathrm{mL})$, a middle concentration group $(0.38 \mathrm{mg} / \mathrm{mL})$, and a low concentration group $(0.19 \mathrm{mg} / \mathrm{mL})$. The cells in the NC group were cultured in RPMI-1640 culture medium containing 10\% FBS. The cells in the LPS group were stimulated with $1000 \mathrm{ng} / \mathrm{mL}$ LPS for 19 hours. The cells in the three YMT-administered groups were pretreated with LPS for 1 hour prior to treatment with different concentration of YMT for 18 hours.

2.4. Human Inflammasomes PCR Array. Human Inflammasomes PCR Array was performed to evaluate the expression of 84 inflammasomes-related genes. Total RNA was extracted from PBMCs using the RNeasy Mini Kit. An UNIC 2800 UV/VIS Spectrophotometer was used to assess the quantity and quality of the RNA extracts by measuring the absorbance at 260 and $280 \mathrm{~nm}$. Total RNA was purified using an RNase-Free DNase Set. cDNA was generated by reverse transcription of $20 \mathrm{ng}$ of total RNA from each sample using the $\mathrm{RT}^{2}$ First Strand Kit and then combined with the $\mathrm{RT}^{2} \mathrm{SYBR}^{\circledR}$ Green ROX qPCR Mastermix in 96 well arrays. Thermal cycling was performed using an ABI Prism SDS 7300 system (Applied Biosystems). Gene expression was compared using the $\mathrm{Ct}$ values and calculated using the $\Delta \Delta \mathrm{Ct}$ method with normalization to the average expression levels of five common genes (ACTB, B2 M, GAPDH, HPRT, and RPL13A) [16].

2.5. Western Blot. After treatment, the cells were collected and lysed in RIPA buffer (Sigma, St. Louis, MO, USA). $25 \mu \mathrm{g}$ protein per lane was loaded onto SDS-PAGE gels and then 


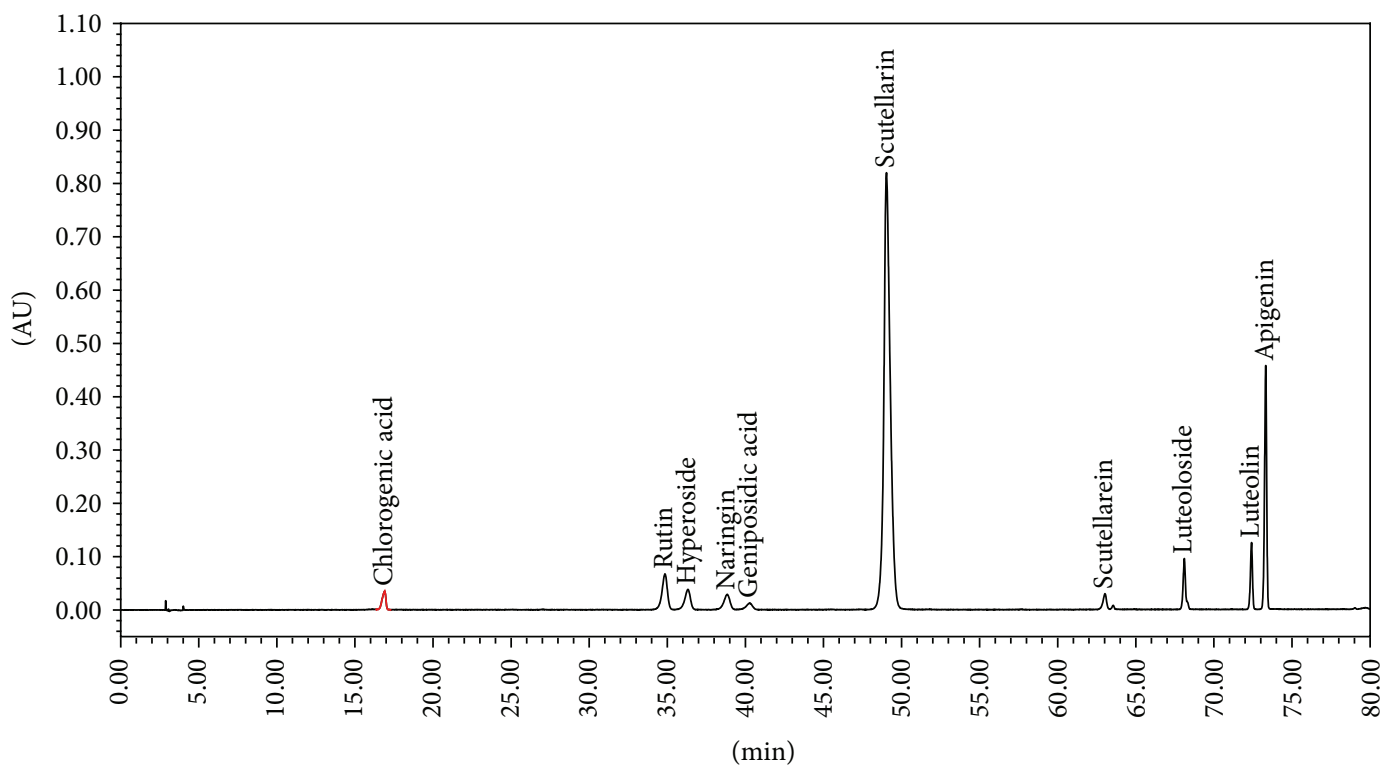

FIGURE 1: The fingerprints of the mixed standard compounds.

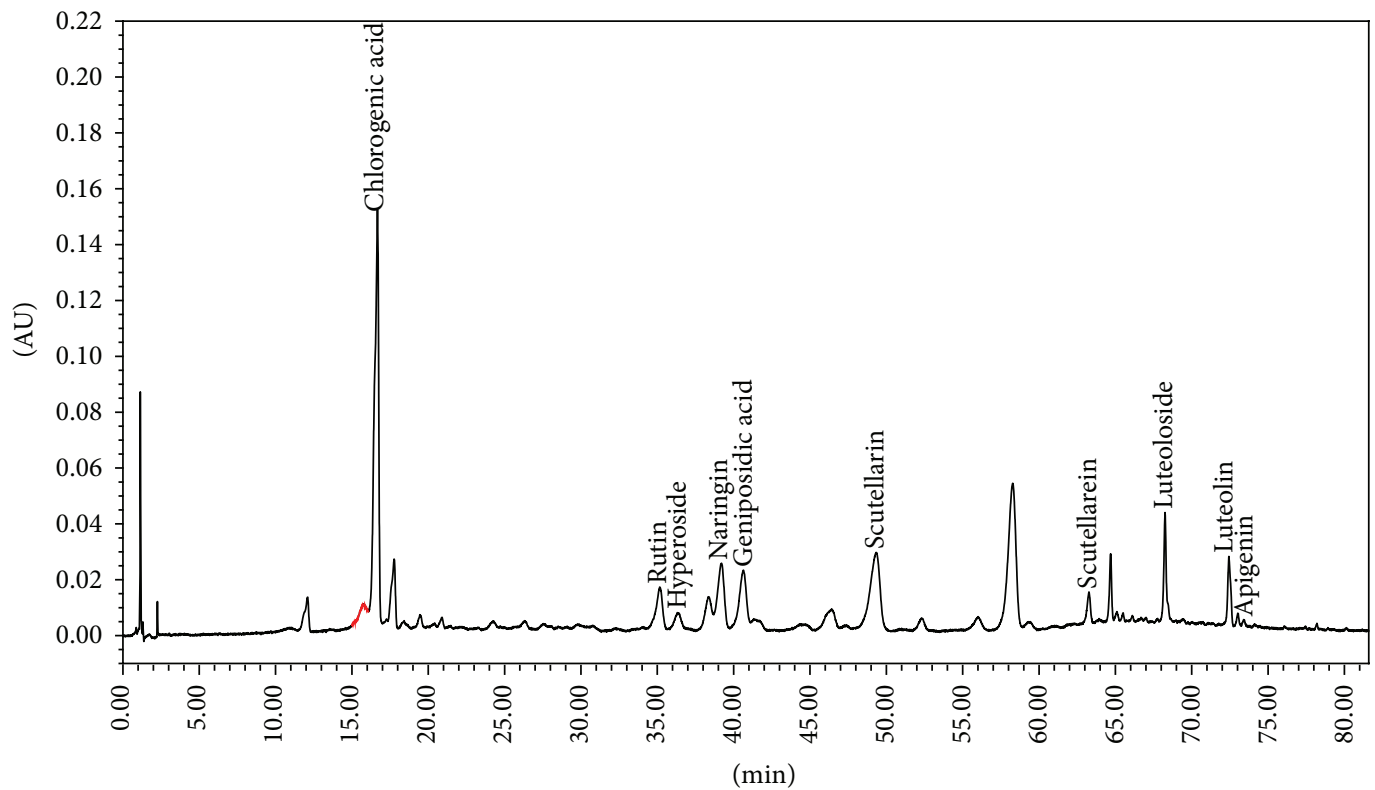

FIGURE 2: The fingerprints of extract of YMT.

were transferred onto nitrocellulose membranes (Millipore Corp., Billerica, MA, USA). The membranes were then blocked and probed using antibodies that were raised against the target proteins, including $\beta$-actin, NLRP3, Caspase-1, and NLRP1, and were incubated with appropriate secondary antibodies for $2 \mathrm{~h}$. A coloration solution mixture (Beyotime, Jiangsu, China) was added and the immunoreactive bands were determined after exposure to the solution.

2.6. Enzyme-Linked Immunosorbent Assay. Protein expression was measured using TNF- $\alpha$, IL-1 $\beta$, IL-6, IL-10, and MCP-1 enzyme-linked immunosorbent assay (ELISA) kits according to manufacturer's instruction.
2.7. Statistical Analyses. The data were presented as the means \pm SD. Statistical analyses of the data were performed using Student's $t$-test. $P<0.05$ was considered significant. Statistical comparisons among three groups were performed using one-way ANOVA. These analyses were performed using SPSS 18.0 software (USA) [17, 18].

\section{Results}

3.1. LPS-Induced Inflammatory Injury in PBMCs. After culture for 18 hours, cell viability of trypan blue stained cell reached 95\% after isolation. Our findings showed that after treatment with LPS for 19 hours, the protein expression 


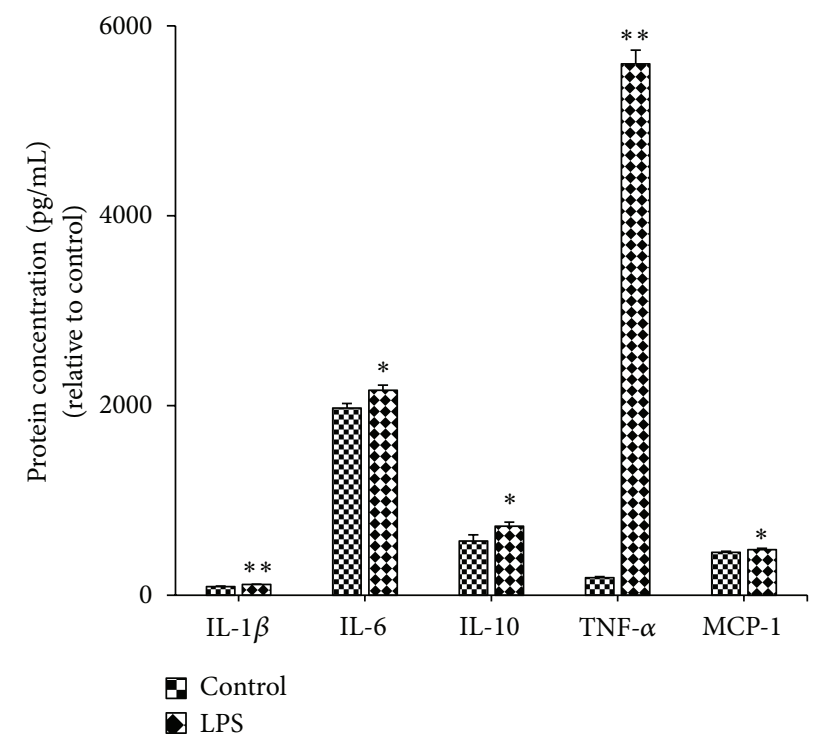

FIgURE 3: Effect of LPS stimulus on the protein production of IL-1 $\beta$, IL-6, IL-10, MCP-1, and TNF- $\alpha$ in PBMC. Control: cells in the NC group were cultured in RPMI-1640 culture medium containing $10 \%$ FBS. LPS: cells in the LPS group were stimulated with $1000 \mathrm{ng} / \mathrm{mL}$ LPS for 19 hours. The protein levels of IL-1 $\beta$, IL-6, IL-10, MCP-1, and TNF- $\alpha$ were determined by ELISA. Results are presented as mean \pm standard deviation (SD). ${ }^{*} P<0.05$ and ${ }^{* *} P<0.01$ compared to the control group $(n=4)$.

of TNF- $\alpha$, IL-1 $\beta$, IL- 6 , IL-10, and MCP-1 was enhanced significantly (Figure 3 ).

3.2. YMT Induces the Differential Regulation of InflammatoryRelated Genes. We examined the expression of 84 inflammatory-related genes in PBMC from the control group, LPS group, and the YMT-treated groups using the Human Inflammasomes PCR Array. After LPS stimulus, 45 genes were detected with significant change compared to the control group, in which 42 (approximately 93.33\%) were upregulated. However, in the YMT groups, we detected 33 differentially expressed genes compared to the LPS group, in which 5 genes were upregulated (Figure 4(a)). 15 genes in the YMT-treated groups (Fold Change > 2) were significantly downregulated by YMT (Table 1). Pathway analysis of differentially expressed genes using DAVID database demonstrated that these genes were involved in NOD-like receptor signaling, RIG-I-like receptor signaling, apoptosis, Toll-like receptor signaling, and cytosolic DNA-sensing (Figure 4(b)).

3.3. Effect of YMT on the Protein Expression Levels of NLRP3 and Caspase-1 in PBMC. To investigate whether YMTmediated anti-inflammatory effect was regulated by NLRP3/ Caspase-1 inflammasome, the levels of the two genes were measured using Western blot. In addition, the level of another differentially expressed gene, NLRP1, was evaluated as well. Figure 5 showed the levels of NLRP3 and Caspase-1 in YMT-treated groups were downregulated in the study when compared to the LPS group. In addition, the decreased level of NLRP1 in dose-dependent manner was not observed in the investigation (Figure 5).

3.4. Effects of YMT on LPS-Induced TNF- $\alpha, I L-1 \beta, I L-6, I L-$ 10, and MCP-1 Production. The suppression of YMT on the production of TNF- $\alpha$, IL-1 $\beta$, IL-6, IL-10, and MCP-1, which may be due to an anti-inflammatory reaction, was determined using ELISA to measure the levels of TNF- $\alpha$, IL-1 $\beta$, IL-6, IL-10, and MCP-1. Treatment with YMT $(0.75$, 0.38 , and $0.19 \mathrm{mg} / \mathrm{mL}$ ) significantly inhibited LPS-induced TNF- $\alpha$, IL-1 $\beta$, IL-6, IL-10, and MCP-1 production in a dosedependent manner $(P<0.05)$ (Figure 6).

\section{Discussion}

The important functional role of NLRP3/Caspase-1 inflammasome in the anti-inflammatory effect of YMT on LPSinduced model cells was confirmed in the present study. Our results showed that LPS induced the production of inflammatory mediators IL- $1 \beta$, TNF- $\alpha$, IL-6, IL-10, and MCP1 as well as the transcription factors NLRP3 and Caspase-1 in vitro and that YMT significantly inhibited the expression of NLRP3, Caspase-1, and the downstream cytokine IL-1 $\beta$ and suppressed the production of inflammatory mediators TNF- $\alpha$, IL-6, IL-10, and MCP-1 in a dose-dependent manner compared to the LPS group $(P<0.01)$.

In previous studies, several herbal drugs comprising Flos Lonicerae or the extract of Flos Lonicerae were reported to exhibit anti-inflammatory property. Kang et al. found that in vivo treatment of ethyl acetate fraction of Flos Lonicerae (GC7101 ) in experimental gastric ulcer model rats exhibited antiinflammatory effect by decreased myeloperoxidase activity, NF- $\kappa \mathrm{B}$ translocation, and inflammatory cytokines mRNA expression [19]. Cheng et al. treated LPS-activated murine RAW264.7 macrophages with absolute ethanol extract of herbal formula containing Lonicerae Japonicae Flos and observed that the inhibitory effect of the extract on the inflammatory mediators was regulated by the NF- $\kappa \mathrm{B}$ and MAPK signaling pathways [20]. Similarly, in another work, Cheng et al. investigated the in vitro anti-inflammatory activity of herbal formula containing Lonicerae Japonicae Flos and concluded that the anti-inflammatory effect was associated with suppression of the IRAK-1/TAK1 and TBK1/IRF3 signaling pathways [21]. Thus, we assumed that YMT may have anti-inflammatory activity as well and then conducted the investigation to confirm it.

LPS stimulation upregulates the expression of the inflammasome related genes, NLRP3, Caspase-1, and IL- $1 \beta$ in PBMC from multiple sclerosis compared to healthy controls [22]. In agreement with this finding, in the current study the results demonstrated the upregulation of NLRP3 and Caspase-1 in PBMC from the LPS group compared to the control group (Figure 5) and showed the increased level of IL- $1 \beta$, IL- 6 , IL-10, MCP-1, and TNF- $\alpha$ compared to the control group (Figure 3 ). Primed bone-marrowderived macrophages (BMDMs) in vitro stimulated with crude extract of LPS produce IL- $1 \beta$ and LPS-induced CD ${ }^{+}$ $\mathrm{T}$ cell/adipocyte secrete inflammatory mediators TNF- $\alpha$, IL6 , and MCP-1 as well as the transcription factor NF- $\kappa \mathrm{B}$ p 65 

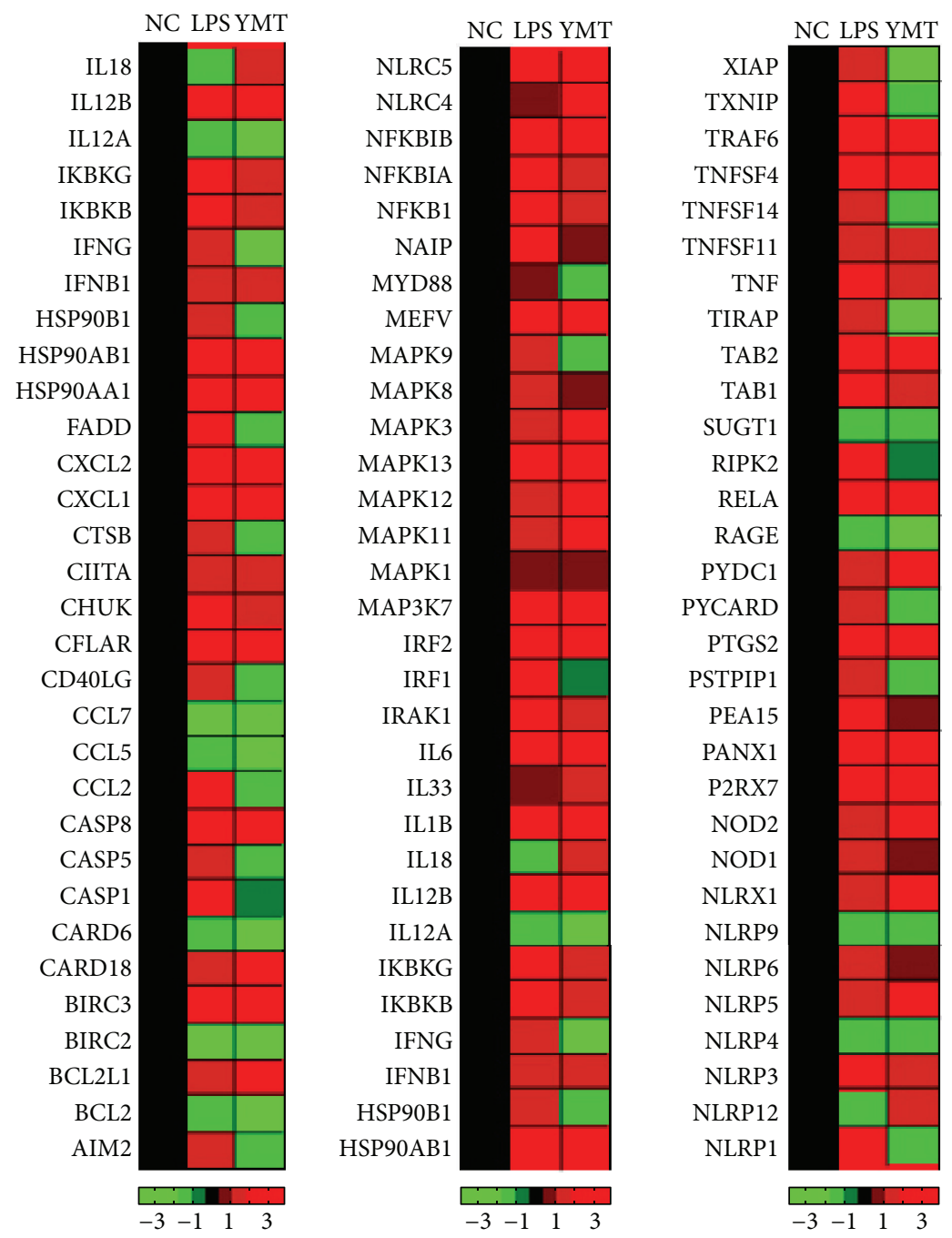

(a)

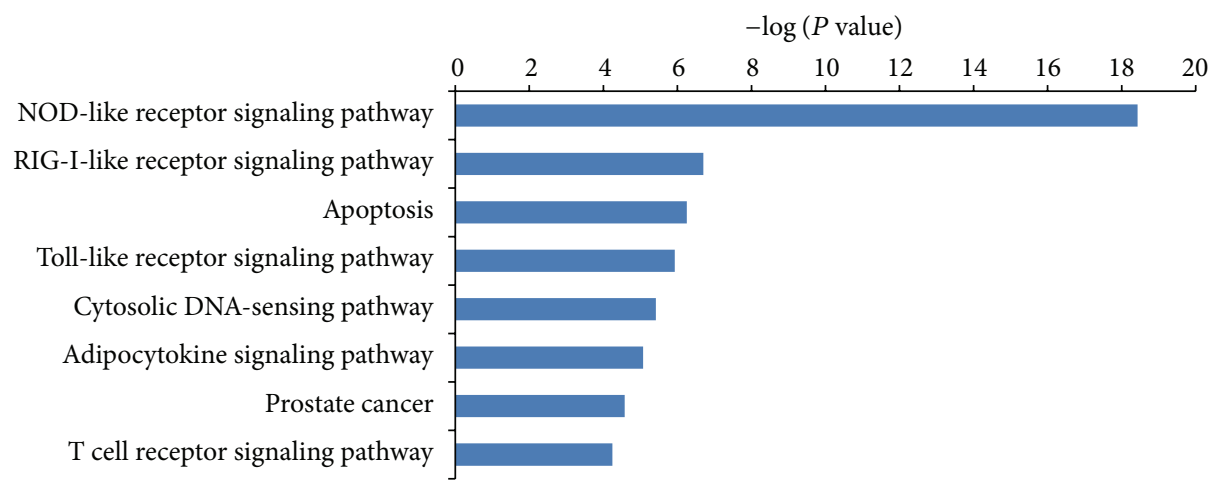

(b)

FIGURE 4: The expression and distribution of differential inflammation-related genes. The results of cluster analysis of 84 inflammationrelated genes (a). NC: cells in the NC group were cultured in RPMI-1640 culture medium containing 10\% FBS. LPS: cells in the LPS group were stimulated with $1000 \mathrm{ng} / \mathrm{mL}$ LPS for 19 hours. YMT: cells in the group were pretreated with LPS for 1 hour prior to the treatment with YMT $(0.75 \mathrm{mg} / \mathrm{mL})$ for 18 hours. Black regions represent the gene expression in the NC group. Red regions represent the elevated gene expression compared to the NC group. Green regions represent the decreased gene expression compared to the NC group. The analysis results of differentially expressed genes in KEGG database (b). 
TABLE 1: Significantly downregulated inflammation-related genes in PBMC following YMT treatment for $18 \mathrm{~h}$.

\begin{tabular}{lcccc}
\hline Number & Gene name & M/N & Y/M & Description \\
\hline 1 & CASP1 & 2.00 & -2.03 & Caspase 1, apoptosis-related cysteine peptidase \\
2 & CCL2 & 2.28 & -2.94 & Chemokine (C-C motif) ligand 2 \\
3 & CHUK & 2.54 & -2.01 & Conserved helix-loop-helix ubiquitous kinase \\
4 & FADD & 2.68 & -4.40 & Fas (TNFRSF6) associated via death domain \\
5 & HSP90AB1 & 5.74 & -2.68 & Heat shock protein 90 kDa alpha, class B member 1 \\
6 & IKBKG & 3.42 & -2.03 & Inhibitor of kappa light polypeptide gene enhancer in B cells, kinase gamma \\
7 & IRF1 & 4.96 & -5.13 & Interferon regulatory factor 1 \\
8 & NFKBIA & 3.65 & -3.33 & Nuclear factor of kappa light polypeptide gene enhancer in B cells inhibitor, alpha \\
9 & NLRP1 & 2.48 & -3.41 & NLR family, pyrin domain containing 1 \\
10 & NLRP3 & 3.13 & -2.17 & NLR family, pyrin domain containing 3 \\
11 & PEA15 & 2.55 & -2.53 & Phosphoprotein enriched in astrocytes 15 \\
12 & RELA & 4.75 & -2.11 & V-rel reticuloendotheliosis viral oncogene homolog A (avian) \\
13 & RIPK2 & 2.37 & -2.44 & Receptor-interacting serine-threonine kinase 2 \\
14 & TNF & 6.06 & -3.65 & Tumor necrosis factor \\
15 & TXNIP & 2.68 & -3.71 & Thioredoxin interacting protein \\
\hline
\end{tabular}

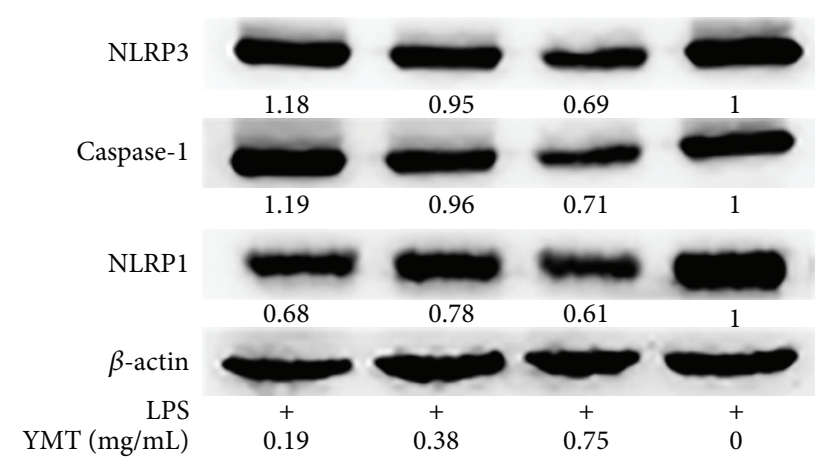

FIGURE 5: Effect of YMT on the protein expression of NLRP3, Caspase-1, and NLRP1 in PBMC. Cells were pretreated with $1000 \mathrm{ng} / \mathrm{mL}$ LPS for 1 hour prior to treatment with different concentration of YMT $(0.19,0.38$, and $0.75 \mathrm{mg} / \mathrm{mL})$ for 18 hours. The last group of cells was stimulated with $1000 \mathrm{ng} / \mathrm{mL}$ LPS for 1 hour. The proteins were extracted from the cell lysates after treatment. Western blot analyses were performed using NLRP3, Caspase-1, and NLRP1 antibodies. $\beta$-actin served as the housekeeping control.

$[23,24]$. After intraperitoneal injection in C57BL/6 mice with LPS for $6 \mathrm{~h}, \mathrm{IL}-1 \beta$ in peritoneal washes was detected, which confirms the in vitro work [23]. LPS, the bacterial endotoxin, binds to the receptor Toll-like receptor 4 (TLR4) and initiates the recruitment of the adaptor myeloid differentiation factor 88 (MyD88) to Toll-interleukin 1 resistance (TIR) domain, resulting in the activation of multiple signaling pathways and the production of proinflammatory mediators [25]. Thus, we chose LPS and PBMC to make inflammatory model to investigate the anti-inflammatory effect of YMT in vitro.

We observed the LPS-induced mRNA and protein expression of NLRP3 and Caspase-1, the two components of the NLRP3 inflammasome, was markedly downregulated after YMT treatment in PBMC (Figure 5, Table 1). Oh et al. discovered that the anti-inflammatory activity of methanol extract of Morus bombycis Koidzumi was mediated by attenuation of NLRP3 inflammasome activation and regulation of the interferon- $\beta$ receptor signaling pathway in LPS-stimulated RAW264.7 cells and bone-marrow-derived murine macrophages [26]. Sun et al. observed that Impatiens textori Miq. extract exhibited the anti-inflammatory effect via inhibition of NLRP3 inflammasome activation in in vitro and in vivo experimental models and observed decreased amount of Caspase-1 maturation as well [27]. NLRP1 activation leads to the recruitment and activation of the downstream protease Caspase-1 and the secretion of IL-1 $\beta$ and IL-18 [28]. Moreover, we did not discover the decreased protein production of NLRP1 despite the suppressed mRNA level after YMT treatment for $18 \mathrm{~h}$ (Figure 5). Thus, we concluded that NLRP1 was not involved in antiinflammatory mechanism of YMT and that the inhibition of NLRP3/Caspase inflammasome was responsible for antiinflammatory effect of YMT in vitro. The NLRP3 inflammasome, including NLRP3, ASC, and Caspase-1, accumulates at the inflammatory sites and activates inactive pro-Caspase-1 when exogenous or endogenous danger signals are detected. Active Caspase-1 maturates the two inflammatory mediators pro-IL-1 $\beta$ and pro-IL-18 by proteolysis and promotes their secretion. Via KEGG database analysis, we found that YMT mainly mediated NOD-like receptor signaling pathway and Toll-like receptor signaling pathway, indicating that YMT exerted strong anti-inflammatory activities by enhancing innate immune responses. It is known that binding of LPS to TLR4 activates several intracellular signaling pathways that include the I $\kappa \mathrm{B}$ kinase- (IKK-) NF- $\kappa \mathrm{B}$ pathway and MAPK pathways, resulting in the expression of IL- $1 \beta$, IL-18, TNF- $\alpha$, and NLRP3 [29]. We noted the downregulation of five NF$\kappa \mathrm{B}$ related genes including RIPK2, RELA, IKBKG, NFKBIA, and CHUK. Among them, RIPK2, RELA, and IKBKG are responsible for encoding and activating the transcription factor, NF- $\kappa \mathrm{B}$, and in contrast NFKBIA and CHUK inhibit the expression of NF- $\kappa$ B. In previous studies, the inhibition of $N F-\kappa B$ signaling pathway was reported to play an important role in the regulation of anti-inflammatory effect 


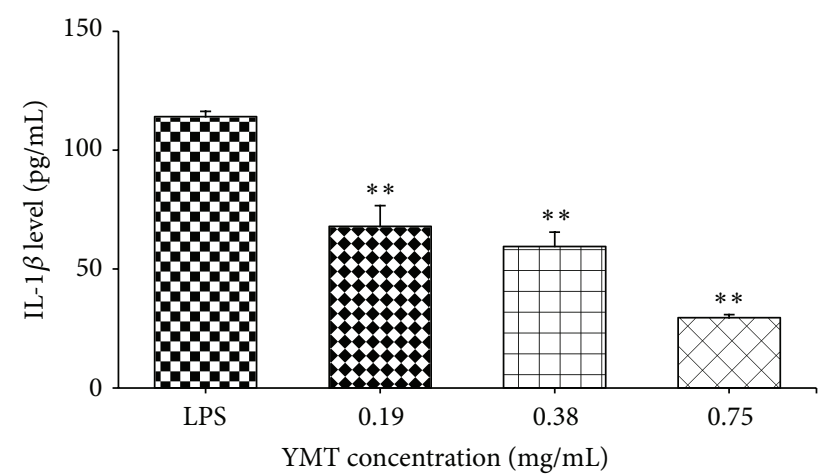

(a)

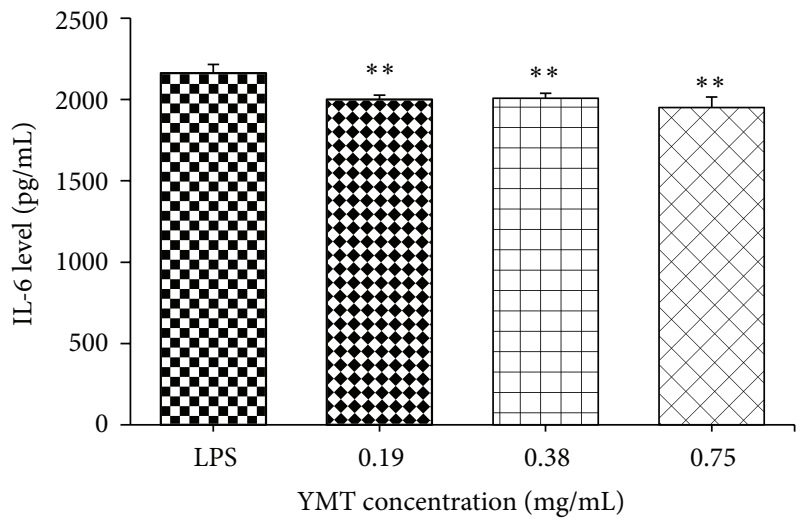

(c)

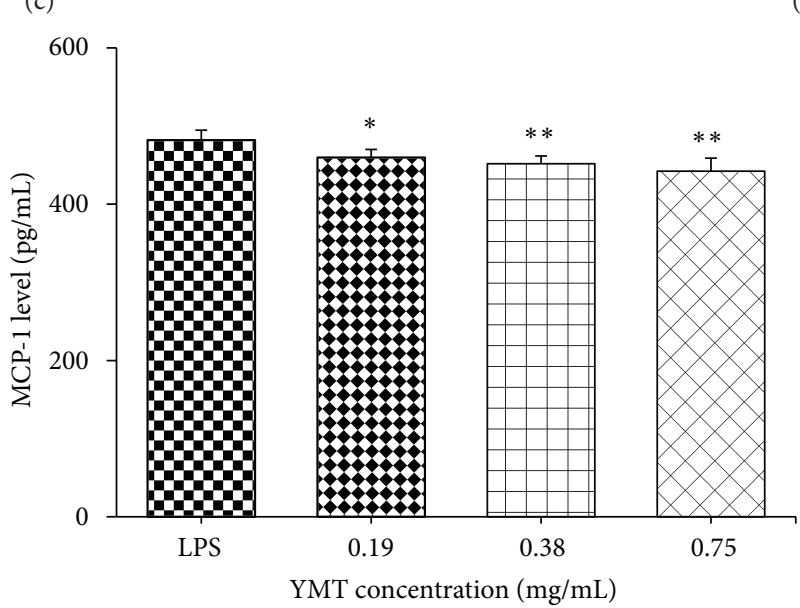

(e)

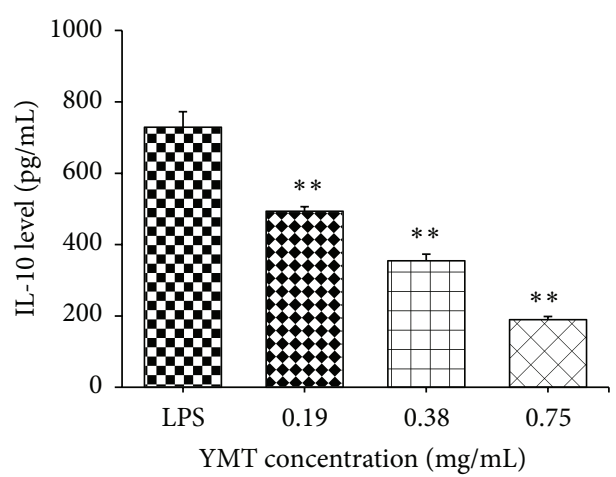

(b)

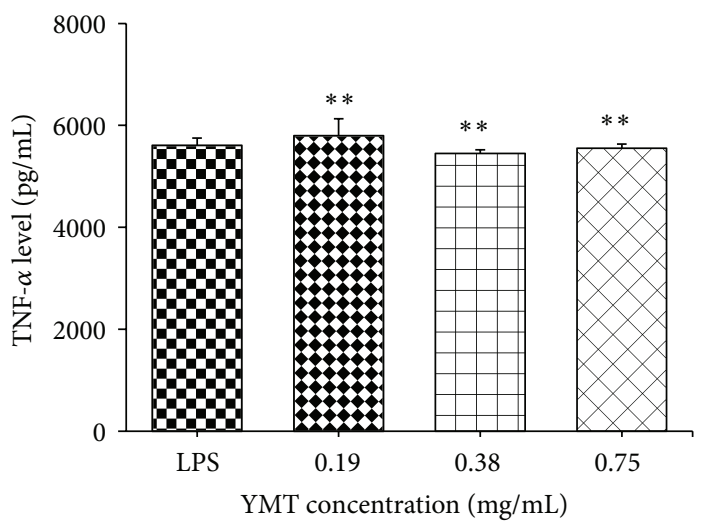

(d) 
than function solely, which forms a negative feedback loop and thus enhances innate immunity. IL-1 $\beta$, a downstream protein in the NLRP3/Caspase-1 pathway, cooperates with other cytokines to activate $\mathrm{B}$ cells and $\mathrm{T}$ cells, induce the production of other inflammatory mediators including TNF$\alpha$ and IL- 6 , and strengthen the adhesion of leucocytes to endothelial cells. To verify the results obtained in PCR array, we conducted ELISA assay to determine the protein level of inflammation-related genes. The protein production of IL$1 \beta$, TNF- $\alpha$, IL- 6 , IL-10, and MCP-1 in supernatant of culture medium decreased significantly after YMT pretreatment in the study $(P<0.01)$ (Figure 6$)$. Under the LPS stimulation, mononuclear macrophage and other inflammatory cells are irritated to produce and release large amount of multiple inflammatory mediators [34], which is consistent with our finding.

\section{Conclusions}

In summary, YMT protects against LPS-induced inflammation in PBMC through the inhibition of the NLRP3/Caspase inflammasome, reducing the production of IL-1 $\beta$, TNF$\alpha$, IL-6, IL-10, and MCP-1 and suppressing the inflammatory response. The findings demonstrated the strong antiinflammatory properties in vitro and inhibitory mechanism of YMT in NLRP3/Caspase inflammasome. Therefore, the anti-inflammatory ability of YMT may have therapeutic potential for the treatment of other inflammatory diseases in addition to acute pyelonephritis, acute urocystitis, and chronic prostatitis.

\section{Competing Interests}

The authors declare that there are no competing interests regarding the publication of this paper.

\section{Authors' Contributions}

Jingying Sai and Lingxin Xiong contributed equally to the work.

\section{Acknowledgments}

The project was supported by Jilin Provincial Science and Technology Planning Program (Grant no. 20150519015JH) and project supported by the Association of Young Scientists and Technologists of Jilin University (Grant no. 450060525011).

\section{References}

[1] X. Zhang, C. Zhang, J. Sai et al., "Xueshuan xinmaining tablet treats blood stasis through regulating the expression of F13a1, Carl, and Tbxa2r," Evidence-Based Complementary and Alternative Medicine, vol. 2015, Article ID 704390, 8 pages, 2015.

[2] The State Pharmacopeia Commission of the People's Republic of China, Pharmacopoeia of of the People's Republic of China, China Medical Science Press, Beijing, China, 2015.
[3] L. Reeve, H. Lashen, and A. A. Pacey, "Endometriosis affects sperm-endosalpingeal interactions," Human Reproduction, vol. 20, no. 2, pp. 448-451, 2005.

[4] J. Y. Sai, Y. Hu, C. Zhang et al., "Influence of ethanol extract of Yinhuamiyanling Tablets in ultrastructure of Escherichia coli and its antibacterial mechanism," Journal of Jilin University (Medicine Edition), vol. 40, no. 1, pp. 117-120, 2014.

[5] K. Ohgami, I. Ilieva, K. Shiratori et al., "Anti-inflammatory effects of aronia extract on rat endotoxin-induced uveitis," Investigative Ophthalmology and Visual Science, vol. 46, no. 1, pp. 275-281, 2005.

[6] R. Puupponen-Pimiä, L. Nohynek, C. Meier et al., "Antimicrobial properties of phenolic compounds from berries," Journal of Applied Microbiology, vol. 90, no. 4, pp. 494-507, 2001.

[7] X. F. Zhu, H. X. Zhang, and R. Lo, "Phenolic compounds from the leaf extract of artichoke (Cynara scolymus L.) and their antimicrobial activities," Journal of Agricultural and Food Chemistry, vol. 52, no. 24, pp. 7272-7278, 2004.

[8] F. Jiang and G. J. Dusting, "Natural phenolic compounds as cardiovascular therapeutics: potential role of their antiinflammatory effects," Current Vascular Pharmacology, vol. 1, no. 2, pp. 135-156, 2003.

[9] S. Park, K. I.-B. Hahm, T.-Y. Ho, J.-H. Jin, and R. Choue, "Preventive effect of the flavonoid, wogonin, against ethanolinduced gastric mucosal damage in rats," Digestive Diseases \& Sciences, vol. 49, no. 3, pp. 384-394, 2004.

[10] Y. Takada and B. B. Aggarwal, "Flavopiridol inhibits NF- $\kappa$ B activation induced by various carcinogens and inflammatory agents through inhibition of $\mathrm{I} \kappa \mathrm{B} \alpha$ kinase and p 65 phosphorylation. Abrogation of cyclin D1, cyclooxygenase-2, and matrix metalloprotease-9," The Journal of Biological Chemistry, vol. 279, no. 6, pp. 4750-4759, 2004.

[11] A. Xagorari, A. Papapetropoulos, A. Mauromatis, M. Economou, T. Fotsis, and C. Roussos, "Luteolin inhibits an endotoxin-stimulated phosphorylation cascade and proinflammatory cytokine production in macrophages," Journal of Pharmacology and Experimental Therapeutics, vol. 296, no. 1, pp. 181-187, 2001.

[12] X.-H. Jin, K. Ohgami, K. Shiratori et al., "Effects of blue honeysuckle (Lonicera caerulea L.) extract on lipopolysaccharideinduced inflammation in vitro and in vivo," Experimental Eye Research, vol. 82, no. 5, pp. 860-867, 2006.

[13] W.-C. Chen, S.-S. Liou, T.-F. Tzeng, S.-L. Lee, and I.-M. Liu, "Wound repair and anti-inflammatory potential of Lonicera japonica in excision wound-induced rats," BMC Complementary and Alternative Medicine, vol. 12, article 226, 2012.

[14] R. Medzhitov, "Origin and physiological roles of inflammation," Nature, vol. 454, no. 7203, pp. 428-435, 2008.

[15] G. Yeretssian, "Effector functions of NLRs in the intestine: innate sensing, cell death, and disease," Immunologic Research, vol. 54, no. 1-3, pp. 25-36, 2012.

[16] A. Chen, I. Cuevas, P. A. Kenny et al., "Endothelial cell migration and vascular endothelial growth factor expression are the result of loss of breast tissue polarity," Cancer Research, vol. 69, no. 16, pp. 6721-6729, 2009.

[17] J.-W. Yao, J. Liu, X.-Z. Kong et al., "Induction of activation of the antioxidant response element and stabilization of Nrf2 by 3-(3pyridylmethylidene)-2-indolinone (PMID) confers protection against oxidative stress-induced cell death," Toxicology and Applied Pharmacology, vol. 259, no. 2, pp. 227-235, 2012. 
[18] T.-Y. Wu, C. L.-L. Saw, T. O. Khor, D. Pung, S. S. S. Boyanapalli, and A.-N. T. Kong, "In vivo pharmacodynamics of indole-3carbinol in the inhibition of prostate cancer in transgenic adenocarcinoma of mouse prostate (TRAMP) mice: involvement of Nrf2 and cell cycle/apoptosis signaling pathways," Molecular Carcinogenesis, vol. 51, no. 10, pp. 761-770, 2012.

[19] J.-W. Kang, N. Yun, H.-J. Han, J.-Y. Kim, J.-Y. Kim, and S.M. Lee, "Protective effect of flos lonicerae against experimental gastric ulcers in rats: mechanisms of antioxidant and anti-inflammatory action," Evidence-Based Complementary and Alternative Medicine, vol. 2014, Article ID 596920, 11 pages, 2014.

[20] B. C.-Y. Cheng, X.-Q. Ma, H.-Y. Kwan et al., "A herbal formula consisting of Rosae Multiflorae Fructus and Lonicerae Japonicae Flos inhibits inflammatory mediators in LPS-stimulated RAW 264.7 macrophages," Journal of Ethnopharmacology, vol. 153, no. 3, pp. 922-927, 2014.

[21] B. C. Y. Cheng, H. Yu, T. Su et al., "A herbal formula comprising Rosae Multiflorae Fructus and Lonicerae Japonicae Flos inhibits the production of inflammatory mediators and the IRAK1/TAK1 and TBK1/IRF3 pathways in RAW 264.7 and THP-1 cells," Journal of Ethnopharmacology, vol. 174, pp. 195-199, 2015.

[22] E. Peelen, J. Damoiseaux, A.-H. Muris et al., "Increased inflammasome related gene expression profile in PBMC may facilitate $\mathrm{T}$ helper 17 cell induction in multiple sclerosis," Molecular Immunology, vol. 63, no. 2, pp. 521-529, 2015.

[23] A. Ives, J. Nomura, F. Martinon et al., "Xanthine oxidoreductase regulates macrophage IL1 $\beta$ secretion upon NLRP3 inflammasome activation," Nature Communications, vol. 6, article 6555, 2015.

[24] J. M. Monk, D. M. Liddle, A. A. De Boer et al., "Fish-oil-derived n-3 PUFAs reduce inflammatory and chemotactic adipokinemediated cross-talk between co-cultured murine splenic $\mathrm{CD}^{+}$ T cells and adipocytes," Journal of Nutrition, vol. 145, no. 4, pp. 829-838, 2015.

[25] K. A. Fitzgerald, D. C. Rowe, and D. T. Golenbock, "Endotoxin recognition and signal transduction by the TLR4/MD2complex," Microbes and Infection, vol. 6, no. 15, pp. 1361-1367, 2004.

[26] N.-H. Oh, J.-W. Han, D.-W. Shim et al., "Anti-inflammatory properties of Morus bombycis Koidzumi via inhibiting IFN$\beta$ signaling and NLRP3 inflammasome activation," Journal of Ethnopharmacology, vol. 176, pp. 424-428, 2015.

[27] X. Sun, D.-W. Shim, J.-W. Han et al., "Anti-inflammatory effect of Impatiens textori Miq. extract via inhibition of NLRP3 inflammasome activation in in vitro and in vivo experimental models," Journal of Ethnopharmacology, vol. 170, pp. 81-87, 2015.

[28] F. Martinon, K. Burns, and J. Tschopp, “The inflammasome: a molecular platform triggering activation of inflammatory caspases and processing of proIL- $\beta$," Molecular Cell, vol. 10, no. 2, pp. 417-426, 2002.

[29] T.-T. Huang, S.-P. Wu, K.-Y. Chong et al., "The medicinal fungus Antrodia cinnamomea suppresses inflammation by inhibiting the NLRP3 inflammasome," Journal of Ethnopharmacology, vol. 155, no. 1, pp. 154-164, 2014.

[30] H. Babazada, F. Yamashita, S. Yanamoto, and M. Hashida, "Selfassembling lipid modified glycol-split heparin nanoparticles suppress lipopolysaccharide-induced inflammation through TLR4-NF- $\kappa$ B signaling," Journal of Controlled Release, vol. 194, pp. 332-340, 2014.

[31] C. Guiducci, M. Gong, Z. Xu et al., "TLR recognition of self nucleic acids hampers glucocorticoid activity in lupus," Nature, vol. 465 , no. 7300 , pp. 937-941, 2010.
[32] X.-F. Sun and H. Zhang, "NFKB and NFKBI polymorphisms in relation to susceptibility of tumour and other diseases," Histology \& Histopathology, vol. 22, no. 12, pp. 1387-1398, 2007.

[33] X. M. Mo and H. X. Sun, "The anti-inflammatory effect of the CXCR4 antagonist-N15P peptide and its modulation on inflammation-associated mediators in LPS-induced PBMC," Inflammation, vol. 38, no. 3, pp. 1374-1383, 2015.

[34] H. J. Ko, O. S. Kwon, J. H. Jin, K. H. Son, and H. P. Kim, "Inhibition of experimental systemic inflammation (septic inflammation) and chronic bronchitis by new phytoformula BL containing Broussonetia papyrifera and Lonicera japonica," Biomolecules and Therapeutics, vol. 21, no. 1, pp. 66-71, 2013. 


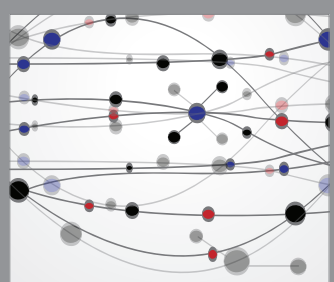

The Scientific World Journal
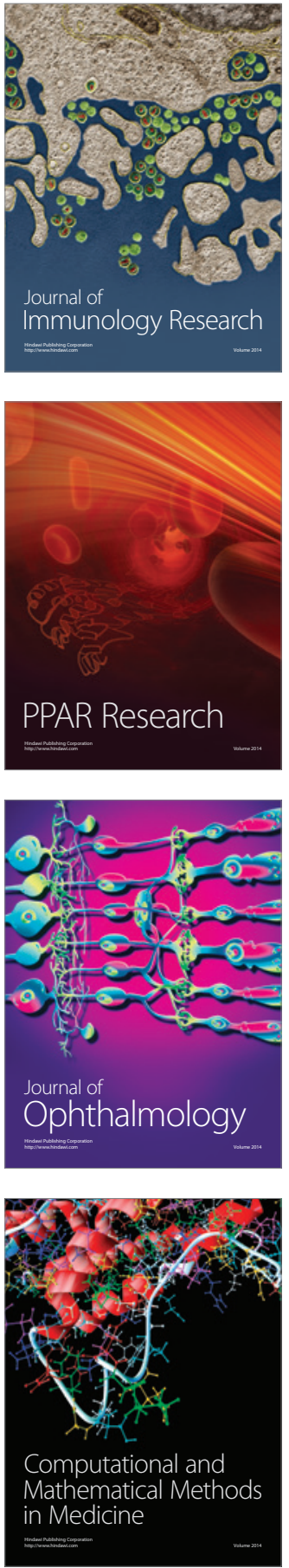

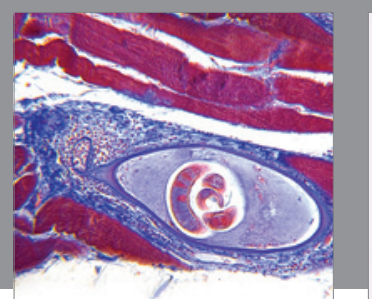

Gastroenterology Research and Practice

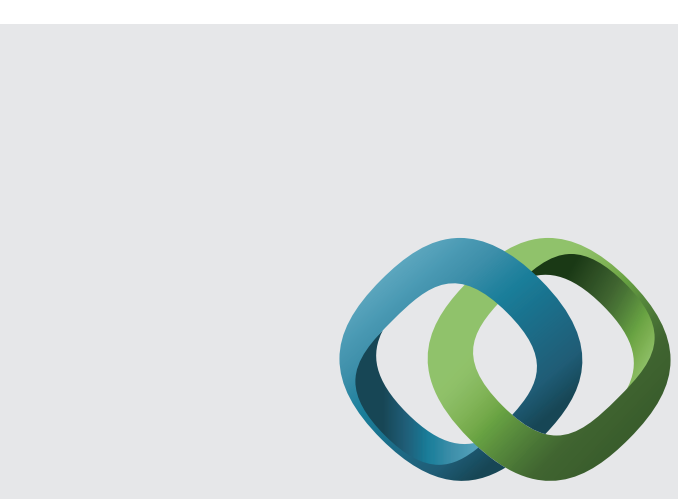

\section{Hindawi}

Submit your manuscripts at

http://www.hindawi.com
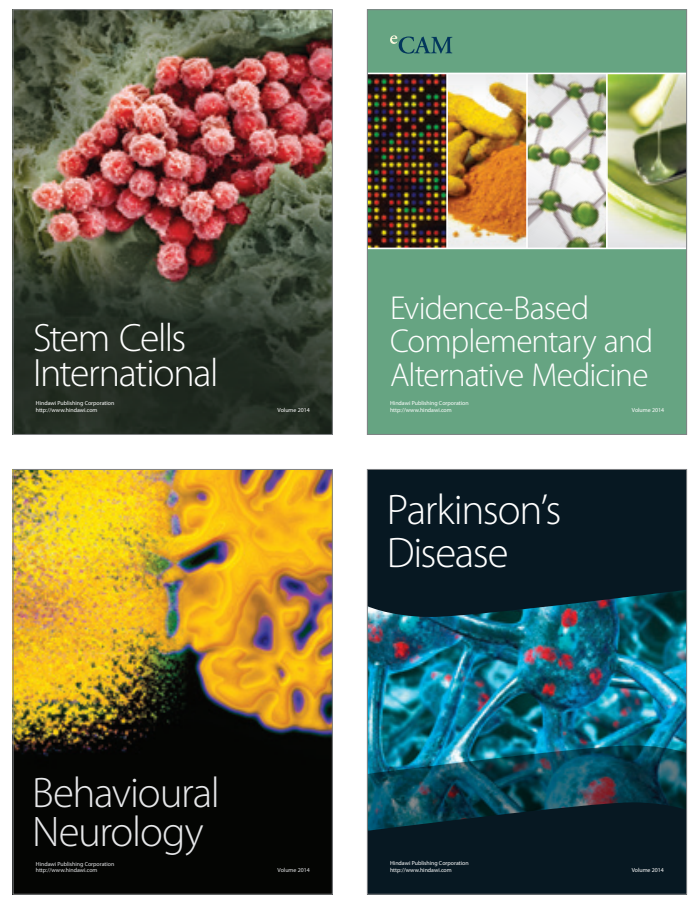
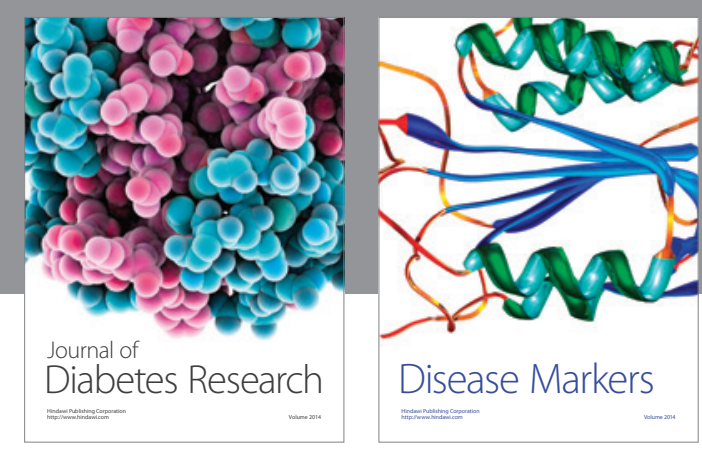

Disease Markers
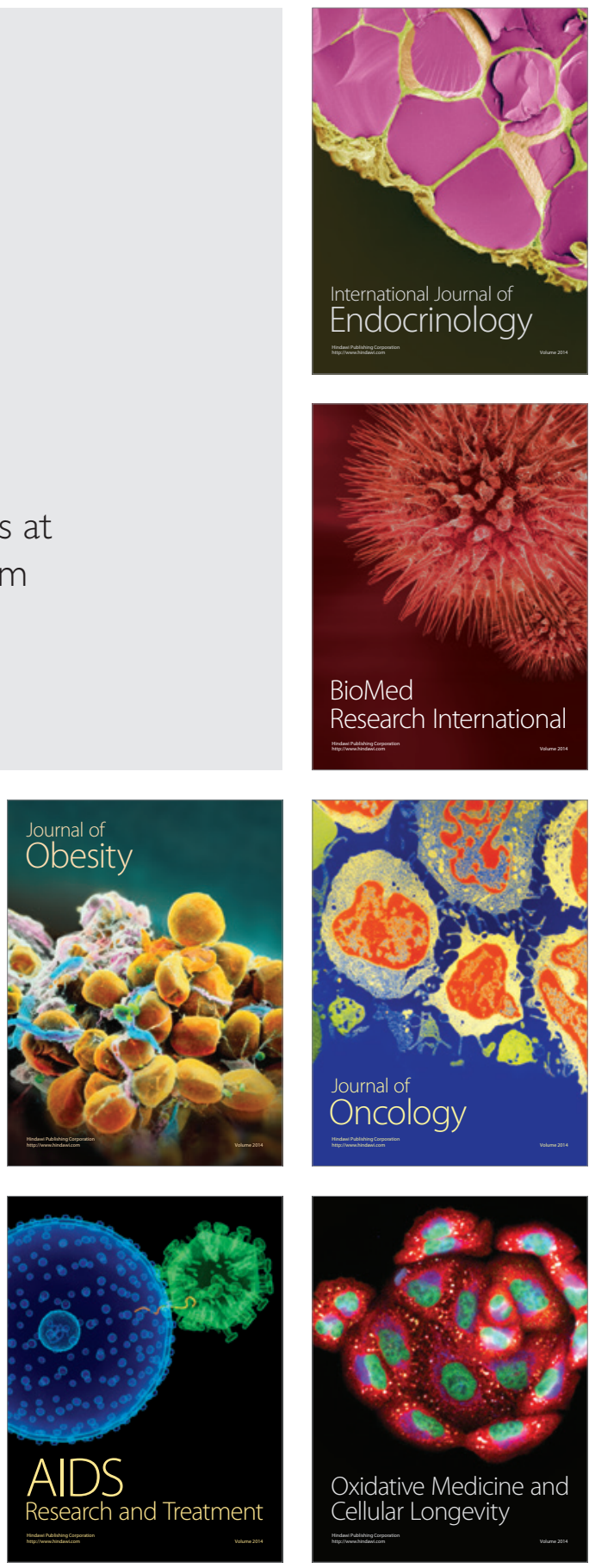